\title{
Prevalence and risk factor analysis of lower extremity abnormal alignment characteristics among rice farmers
}

This article was published in the following Dove Press journal:

Patient Preference and Adherence

17 June 2015

Number of times this article has been viewed

\author{
Usa Karukunchit ${ }^{1,2}$ \\ Rungthip Puntumetakul ${ }^{1,3}$ \\ Manida Swangnetr ${ }^{1,4}$ \\ Rose Boucaut ${ }^{5}$ \\ 'Research Center in Back, Neck, \\ Other Joint Pain and Human \\ Performance (BNOJPH), ${ }^{2}$ Faculty of \\ Associated Medical Sciences, Khon \\ Kaen University, ${ }^{3}$ School of Physical \\ Therapy, Faculty of Associated \\ Medical Sciences, Khon Kaen \\ University, ${ }^{4}$ Department of Production \\ Technology, Faculty of Technology, \\ Khon Kaen University, Khon Kaen, \\ Thailand; ${ }^{5}$ School of Health Sciences \\ (Physiotherapy), iCAHE (International \\ Centre for Allied Health Evidence), \\ Sansom Institute for Health Research, \\ University of South Australia, \\ Adelaide, SA, Australia
}

Background: Rice farming activities involve prolonged manual work and human-machine interaction. Prolonged farming risk-exposure may result in lower limb malalignment. This malalignment may increase the risk of lower extremity injury and physical disabilities. However, the prevalence and factors associated with lower extremity malalignment have not yet been reported. This study aimed to investigate the prevalence and risk factors of lower extremity malalignment among rice farmers.

Methods: A cross-sectional survey was conducted with 249 rice farmers. Lower extremity alignment assessment included: pelvic tilt angle, limb length equality, femoral torsion, quadriceps (Q) angle, tibiofemoral angle, genu recurvatum, rearfoot angle, and medial longitudinal arch angle. Descriptive statistics were used to analyze participant characteristics and prevalence of lower extremity malalignment. Logistic regression analysis was used to identify risk factors.

Results: The highest prevalence of lower extremity malalignment was foot pronation $(36.14 \%)$, followed by the abnormal Q angle (34.94\%), tibiofemoral angle (31.73\%), pelvic tilt angle $(30.52 \%)$, femoral antetorsion $(28.11 \%)$, limb length inequality $(22.49 \%)$, tibial torsion (21.29\%), and genu recurvatum (11.24\%). In females, the risk factors were abnormal Q angle, tibiofemoral angle, and genu recurvatum. Being overweight was a risk factor for abnormal pelvic tilt angle, Q angle, and tibiofemoral angle. Age was a risk factor for limb length inequality. Years of farming were a major risk factor for abnormal $\mathrm{Q}$ angle, tibiofemoral angle, and foot malalignment.

Conclusion: Prevalence of lower extremity malalignment was reported in this study. Female sex, being overweight, and years of farming were major risk factors for lower extremity malalignment. Lower extremity screening should assist in the identification of foot and knee malalignment in rice farmers. This may then lead to early prevention of musculoskeletal disorders arising from such malalignment.

Keywords: lower extremity malalignment, prevalence, rice farmer, risk factors

\section{Introduction}

Rice farming is an important agricultural occupation in Asia, especially in Thailand. Rice is one of the top ten exports from Thailand in terms of value. ${ }^{1}$ In 2014 , more than 10 billion kilograms of rice were exported valued at approximately 174 billion Baht (about US\$5.35 billion). ${ }^{1}$ Typical rice cultivation processes include plowing, seeding, planting, nursing, fertilizing, and harvesting. ${ }^{2}$ In Asian countries, rice farming activities are performed manually in extreme environmental conditions. ${ }^{3}$ Most tasks involve repetitive motion, awkward postures, heavy lifting, prolonged standing, and control of heavy machinery. Farmers go barefoot when working. Plowing is performed using heavy vibrating machinery on slippery and sloping walking surfaces (Figure 1A).,
Correspondence: Rungthip Puntumetakul Research Center in Back, Neck, Other Joint Pain and Human Performance (BNOJPH), 123 Khon Kaen University, Mitraphab Street, Muang District, Khon Kaen 40002, Thailand

Tel +66 834196186

Fax +66 4202399

Email rungthiprt@gmail.com 
The seeding, nursing, and fertilization processes involve heavy carrying and walking on muddy surfaces filled with water (Figure $1 \mathrm{~B}$ and C). ${ }^{2}$ The planting task requires repetitive forward trunk bending and twisting, as well as prolonged standing on mud, in water (Figure 1D). ${ }^{2}$ The harvesting process involves prolonged stooping, and walking on rough soil (Figure 1E). ${ }^{2}$ These ergonomic risk factors can result in chronic musculoskeletal disorders (MSDs), especially of the lower extremities. Lower extremity MSDs in rice farmers are reported to be highly prevalent (up to $41 \%$ ). ${ }^{5}$

Exposure to ergonomic risk factors may lead to abnormal biomechanical function and structural adaptation. ${ }^{6-10}$ Lower extremity malalignment may cause musculoskeletal dysfunction including abnormal joint loading, muscle imbalance, and deviation from neutral alignment. ${ }^{8-10}$ Abnormal lower extremity alignment has been associated with increased risk of lower extremity MSDs, including hip and knee osteoarthritis, ${ }^{11}$ patellofemoral pain syndrome, ${ }^{12}$ anterior cruciate ligament injury, ${ }^{8,13}$ and medial tibial stress syndrome. Lower extremity malalignment can result in leg and foot pain, reduced lower extremity mobility, physical disability, and, consequently, reduced work ability. ${ }^{8,11}$ Lower extremity malalignment has important consequences for health and work.
Individual and work-related factors have been found to be associated with lower extremity MSDs. ${ }^{14-16}$ MSDs resulting from repeated abnormal loading can produce lower extremity malalignment. ${ }^{8-10,17}$ However, the prevalence and risk factors of lower extremity malalignment in rice farmers have not yet been reported.

Although both individual and ergonomic factors may increase the risk of lower extremity malalignment, all rice farmers are exposed to similar ergonomic risks related to their farming activities. These risks include repetitive motion, high forces, awkward postures, and extreme environmental conditions. Additionally, lower extremity malalignment might be influenced by individual factors. Therefore, information on individual factors among rice farmers including sex, age, body mass index (BMI), daily work hours, and years of farming were captured in this study.

Females are generally exposed to a higher risk of lower extremity malalignment due to different anatomical alignment, lower pain thresholds, and lower physical tolerance than males. ${ }^{17-20}$ Previous studies found that females are at increased risk of abnormal anterior pelvic tilt, femoral antetorsion, Q angle, tibiofemoral malalignment, and genu recurvatum. ${ }^{17,18}$ However, the sex difference for limb
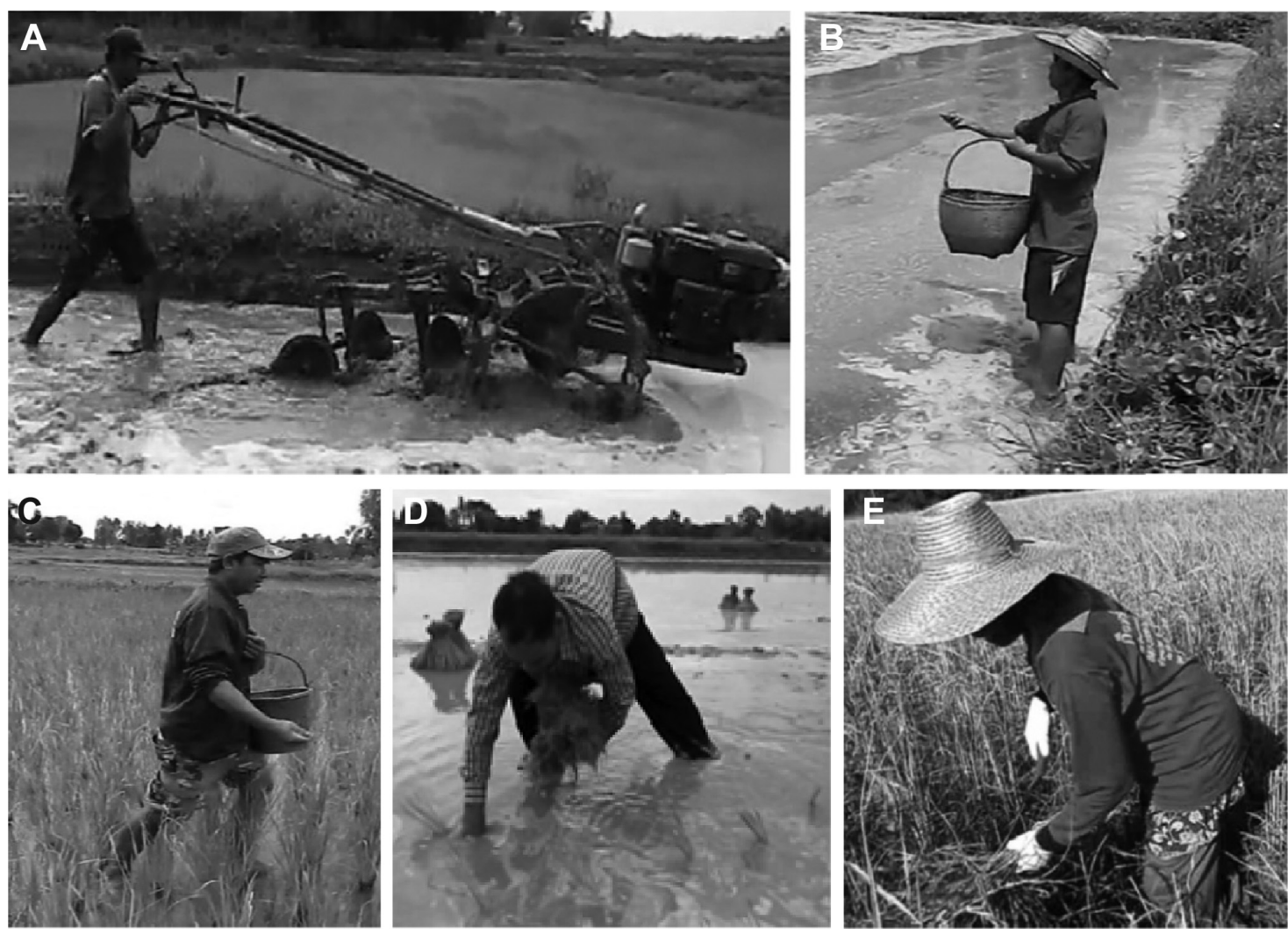

Figure I Typical rice cultivation processes including: (A) plowing; (B) seeding;(C) nursing and fertilizing; (D) planting; and (E) harvesting. 
length inequality and foot pronation have not yet been reported.

Being overweight $\left(\mathrm{BMI} \geq 25 \mathrm{~kg} / \mathrm{m}^{2}\right.$ ) has been reported as a risk factor for lower extremity malalignment, particularly abnormal pelvic tilt, $\mathrm{Q}$ angle, and tibiofemoral angle. This is because increased joint loading can produce injury to weight-bearing joints in the lower limbs. ${ }^{15,16,21}$ Obese persons may compensate for excessive loading by abnormal lower extremity alignment. ${ }^{16}$ Increasing age is associated with degenerative joint conditions, decreased muscle strength, physical performance, and ligament laxity, which may result in lower extremity anatomical adaptations. ${ }^{15,16,19-24}$ Adults aged 40 years and older show positive signs correlated with hip and knee malalignment such as osteoarthritis (OA). ${ }^{14,15,21}$

In addition to these factors, prolonged daily working hours and farming years worked have been identified as risk factors for lower extremity MSDs. ${ }^{14,15}$ Farming activities performed with high ergonomic risk factors in extreme environmental conditions may increase degenerative joint conditions and make it difficult for farmers to maintain good or neutral postures, resulting in lower extremity alignment maladaptation. ${ }^{6,7,23}$ Moreover, experience in farming may be associated with leg pain during weight-bearing activities in muddy soil. ${ }^{4}$

Prevalence and risk factors of lower extremity malalignment have not been reported. Therefore, the objective of this study was to determine the prevalence of, and to identify risk factors associated with, lower extremity malalignment in rice farmers. It is important to understand risk factors associated with lower extremity malalignment in order to develop guidelines for prevention. We hypothesized that sex, age, BMI, daily working hours, and years of farming would be risk factors of lower extremity malalignment. We also hypothesized that sex would be a statistically significant risk factor for lower extremity malalignment, with females demonstrating greater prevalence than males.

We hypothesized that older farmers would demonstrate increased risk of lower extremity malalignment than younger ones. We also expected that being overweight would be a risk factor for lower extremity malalignment. Additionally, we hypothesized that farmers with a higher number of daily working hours and years of farming would be more likely to have lower extremity malalignment than those who worked less.

\section{Methods}

\section{Study design and participants}

This study used a cross-sectional design, and involved rice farmers in the Khon Kaen province, Thailand. The Khon
Kaen Provincial Agricultural Extension Office provided the investigators with a list of rice farmers from their database. Participants (farmers) were included if they were employed in rice farming in the Khon Kaen province and had been rice farming for at least 1 year. Participants were selected by multistage random sampling. Cluster sampling was used to randomly select 18 districts and 267 villages in the Khon Kaen province, and then simple random sampling in a field survey was used to include 249 farmers.

Participants were all primarily rice farmers, with no secondary work. They were aged between 18 and 60 years. Participants were excluded if they reported current symptoms or signs of lower limb injury or any previous history related to lower limb alignment, such as fracture and/or surgery.

The study was conducted at the completion of the rice cultivation process and was approved by the Khon Kaen University Human Ethics Committee before it commenced. Farmers were asked to read and sign a consent form prior to participation in the study. Participants were asked to answer a questionnaire (which took approximately 10 minutes), and were physically assessed to measure lower extremity alignment (approximately 20 minutes/person).

Data collection took place in the Khon Kaen province, Thailand, from October to December 2014. Participants received 250 Baht (US\$7.6) for their participation, which is roughly equivalent to 6 hours of rice farming work. The self-administered questionnaire response rate was $100 \%$ $(n=251)$, but two farmers had a previous history of lower extremity surgery and were therefore excluded from the study. The response proportion of the participants was $99.2 \%$ $(n=249)$. Each of the 249 rice farmers completed the survey, and each was physically assessed for lower extremity alignment. Demographic data including sex, age, BMI, average number of working hours per day, and years of working was collected in the survey. Physical assessment of lower extremity alignment characteristics was undertaken and recorded for each participant.

\section{Measurement of lower extremity alignment}

Lower extremity alignment characteristics were examined to assess the following: limb length inequality, pelvic angle, femoral antetorsion, quadriceps (Q) angle, tibiofemoral angle, genu recurvatum, tibial torsion, rearfoot angle, and medial longitudinal arch angle. All measurements were repeated three times by a single examiner who had excellent intrarater reliability on all lower extremity measures $\left(\mathrm{ICC}_{3,1}\right.$ range of 0.89-0.98). The intrarater reliability was calculated 
from each lower extremity malalignment measurements for 10 subjects. Examiner conducted a second time within 2 days of the first time of measurements on each subject. Lower extremity alignment examinations were conducted as described in the following paragraphs.

Limb length equality was measured using an indirect clinical method. In standing, the greater trochanters and anterior superior iliac spines (ASIS) were palpated to assess limb length. Standard masonite boards ( $3.18 \mathrm{~mm}$ each) were placed under the shorter lower limb, as appropriate. There was no limb length inequality if the greater trochanter and ASIS levels were equal, but inequality was recorded as present if there was a difference of greater than $6.4 \mathrm{~mm}$ (Figure 2A). ${ }^{24}$

Pelvic angle was measured by the angle between a line from the ASIS and posterior superior iliac spines (PSIS) in the horizontal plane using a PALM inclinometer (Performance Attainment Associates, St Paul, MN, USA). ${ }^{25}$ Normal pelvic angle ranges are between $7^{\circ}$ and $15^{\circ} \cdot{ }^{26}$ Abnormal pelvic tilt or anterior pelvic tilt was recorded as present when the ASIS level was lower than the PSIS with more than $15^{\circ}$ tilt (Figure 2B). ${ }^{27,28}$

The femoral antetorsion angle was measured using Craig's test in the prone position with the knee flexed to $90^{\circ}{ }^{29}$ The femur was moved into internal rotation until the greater trochanter was in its maximum lateral position. The angle was measured between a true vertical line and the shaft of the tibia (Figure 2C). Normal femoral antetorsion value ranges from $8^{\circ}$ to $15^{\circ}$. Excessive femoral antetorsion was classified as an angle greater than $30^{\circ} .{ }^{10,26}$

The Q angle is the angle between a line from the ASIS to the center of the patella and a line from the center of the patella to the tibial tuberosity (Figure 2D). ${ }^{29}$ The normal angles for males and females are approximately $10^{\circ}-13^{\circ}$ and $15^{\circ}-18^{\circ}$ respectively. A $\mathrm{Q}$ angle of greater than $18^{\circ}$ was classified as abnormal. ${ }^{26}$

The tibiofemoral angle is formed between a line from the anatomical axis of the femur (midpoint between ASIS and greater trochanter) to the center of the knee and a line from the center of the knee to the center of the ankle (midpoint between medial and lateral malleolar distance) (Figure 2E). ${ }^{29}$ A normal tibiofemoral angle is between $173^{\circ}$ and $180^{\circ}$. An abnormal tibiofemoral angle was less than $173^{\circ} .{ }^{10}$

The test for genu recurvatum was undertaken in a supine position. A bolster was placed under the distal tibia. The knee was moved passively to extend the knee until firm resistance was felt. The angle was measured between a line connecting the femur and the tibia and the sagittal plane
(Figure 2F). ${ }^{29}$ An abnormal genu recurvatum angle value was greater than $10^{\circ} .{ }^{30}$

Tibial torsion testing was undertaken in the supine position with knees extended. The femur was passively rotated to move the femoral epicondyles parallel to the horizontal plane. The angle was represented by a line between the true vertical and a line bisecting the medial and lateral malleoli (Figure 2G). ${ }^{29}$ An angle of more than $40^{\circ}$ represented an abnormal tibial torsion condition. ${ }^{31}$

Rearfoot and medial longitudinal arch angles were measured on the basis of Jonson and Gross's method. ${ }^{32}$ Rearfoot angle was represented by the angle between the line bisecting the calcaneus and the line bisecting the distal one-third of the leg (Figure $2 \mathrm{H}$ ). The medial longitudinal arch angle was measured as the angle between a line from the medial malleolus to the navicular tuberosity and a line from the navicular tuberosity to the medial aspect of the first metatarsal head (Figure 2I). Abnormal foot alignment was identified if the rearfoot angle was greater than $9^{\circ}$ and the medial longitudinal arch angle was less than $134^{\circ} .{ }^{32}$

\section{Statistical analysis}

Descriptive statistics were used to analyze characteristics of participants and lower extremity alignment variables. Continuous variables, including age, average working hours per day, and years working, were analyzed by mean and standard deviation (SD). Categorical variables, including sex, BMI, and lower extremity malalignment characteristics, were considered in terms of frequency and percentage. Simple logistic regression analysis was used to include each independent variable into a multiple logistic regression model. The variables with a $P$-value less than 0.2 were included in the multiple logistic regression model. Multiple logistic regression analysis was performed using a stepwise regression process. The variables with $P$-value less than 0.05 were considered to be statistically significant. ${ }^{33}$ Data were analyzed using the STATA program version 10 (STATA, College Station, TX, USA).

\section{Results}

\section{Characteristics of participants}

Demographic characteristics of the study population are presented in Table 1. There were similar numbers of male and female participants in this study. Most participants $(70.28 \%)$ had a BMI value in the normal range. Average age of participants was 45 years $(\mathrm{SD}=9.29)$. Farmers worked an average of 5.9 hours a day $(\mathrm{SD}=1.21)$. The mean duration of rice farming experience was 25 years $(\mathrm{SD}=11.34)$. 

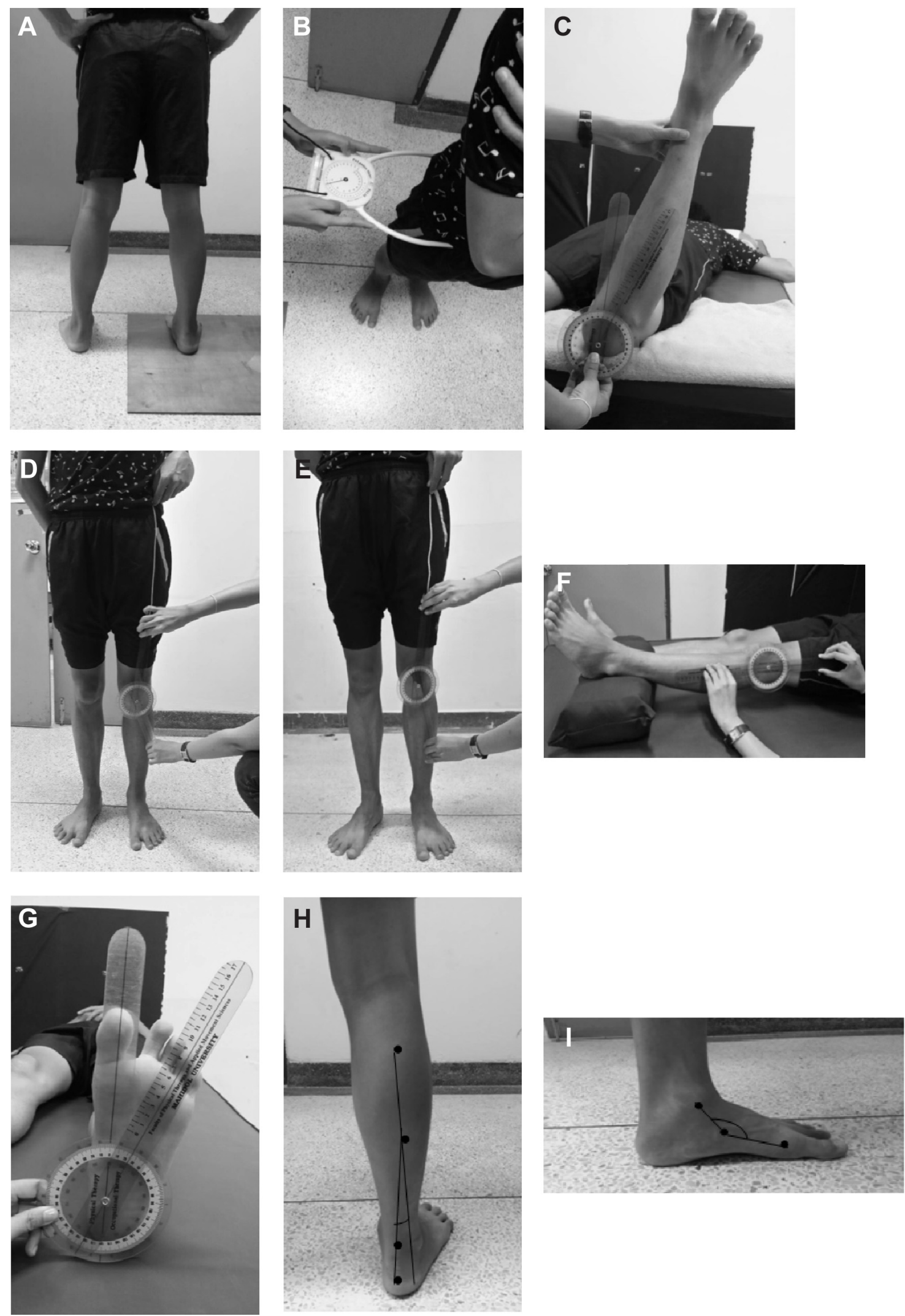

Figure 2 Lower extremity alignment measurement methods for: (A) limb length inequality; (B) pelvic angle; (C) femoral antetorsion angle; (D) $Q$ angle; (E) tibiofemoral angle; (F) genu recurvatum angle; (G) tibial torsion angle; (H) rearfoot angle and; (I) medial longitudinal angle.

Abbreviation: $Q$ angle, quadriceps angle. 
Table I Characteristics of study population $(\mathrm{N}=249)$

\begin{tabular}{|c|c|c|c|}
\hline Characteristics & $\mathbf{N}(\%)$ & Mean \pm SD & Min-max \\
\hline \multicolumn{4}{|l|}{ Sex } \\
\hline Female & $142(57.03)$ & & \\
\hline Male & $107(42.97)$ & & \\
\hline BMI $\left(\mathrm{kg} / \mathrm{m}^{2}\right)$ & & & $16-32.47$ \\
\hline Normal & $175(70.28)$ & & \\
\hline Overweight ( $\left.\geq 25 \mathrm{~kg} / \mathrm{m}^{2}\right)$ & $74(29.72)$ & & \\
\hline Age (years) & & $45.22 \pm 9.29$ & $19-59$ \\
\hline $\begin{array}{l}\text { Daily working hours } \\
\text { (hours/day) }\end{array}$ & & $5.92 \pm 1.21$ & $3-10$ \\
\hline Experience (years) & & $25.67 \pm \mid 1.34$ & $1-46$ \\
\hline
\end{tabular}

Abbreviations: BMI, body mass index; Min-max, minimum-maximum; SD, standard deviation.

\section{Prevalence and risk factors of lower extremity malalignment}

The prevalence of lower extremity malalignment characteristics of rice farmers is presented in Table 2 . The prevalence of malalignment was highest in standing foot alignment (36.14\%), followed by the $\mathrm{Q}$ angle, tibiofemoral angle, pelvic tilt angle, femoral antetorsion, limb length inequality, tibial torsion, and least in genu recurvatum.

\section{Relationship between lower extremity malalignment and risk factors}

The crude odds ratio for univariate logistic regression and the adjusted odds ratio for multiple logistic regression between lower extremity malalignment and risk factors are shown in Tables 3 and 4, respectively.

Female sex was associated with abnormal Q angle, tibiofemoral angle, and genu recurvatum. In addition, females were almost 8.6 times more likely to present with abnormal genu recurvatum compared with males. Being female had a significant and negative effect on femoral antetorsion. Being overweight (BMI $\geq 25 \mathrm{~kg} / \mathrm{m}^{2}$ ) was a statistically significant (increased) risk factor for having abnormal pelvic tilt angle, $\mathrm{Q}$ angle, and tibiofemoral angle. Moreover, those overweight were 8.6 times more likely to demonstrate abnormal anterior

Table 2 Prevalence of lower extremity malalignment in rice farmers

\begin{tabular}{lll}
\hline Characteristics & $\mathbf{N}$ & $\%$ \\
\hline Limb length inequality & 56 & 22.49 \\
Pelvic tilt angle & 76 & 30.52 \\
Femoral antetorsion & 70 & 28.11 \\
Q angle & 87 & 34.94 \\
Tibiofemoral angle & 79 & 31.73 \\
Tibial torsion & 53 & 21.29 \\
Genu recurvatum & 28 & 11.24 \\
Foot pronation & 90 & 36.14 \\
\hline
\end{tabular}

Abbreviation: $Q$ angle, quadriceps angle.

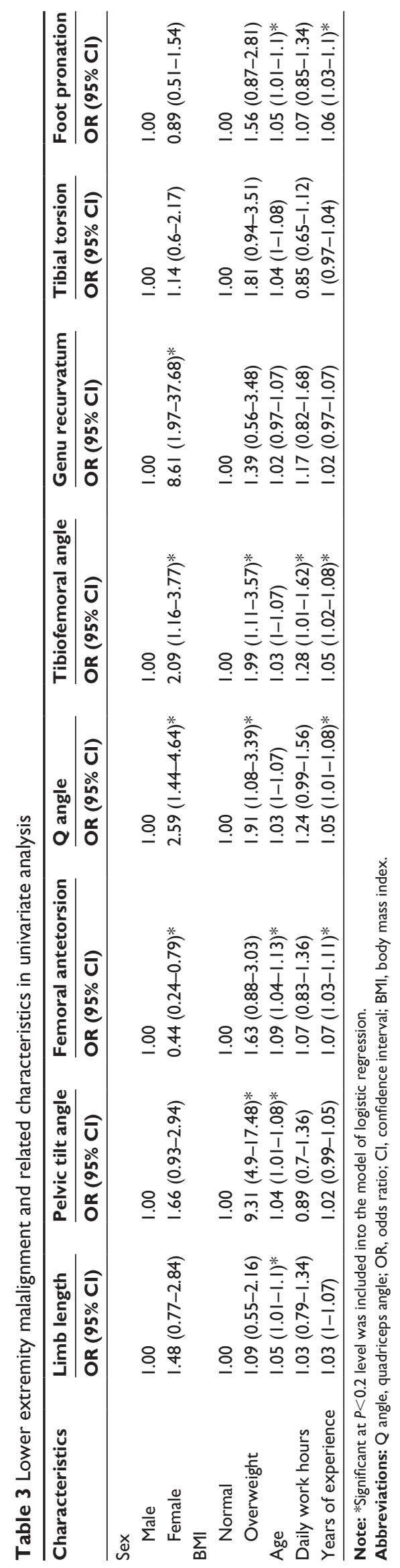


pelvic tilt compared with normal. Increased age was associated with increased risk of having limb length inequality. Farming experience was associated with abnormal Q angle, tibiofemoral angle, and foot alignment. Every increase of 1 year of farming work increased the odds ratio for abnormal Q angle, tibiofemoral angle, and foot alignment ranging from $4 \%$ to $6 \%$.

\section{Discussion}

This study is the first to describe the prevalence and identify risk factors of lower extremity malalignment among Thai rice farmers. Foot and knee malalignment were frequently identified among our sample of rice farmers. The highest prevalence of lower extremity malalignment was foot pronation. Other lower extremity malalignment included abnormal: Q angle, tibiofemoral angle, pelvic tilt angle, femoral antetorsion, limb length inequality, tibial torsion, and genu recurvatum. Limb length inequality can be caused by abnormal lower extremity alignment. Anterior pelvic tilt indicates an anterior rotation of the ilium on the sacrum, and has an effect on lumbar lordosis. Pathology of the pelvic region may result in gait problems. Femoral antetorsion was measured as forward torsion of the femoral neck by the angle between the femoral neck and the condyle. Q angle was assessed to evaluate the knee joint, particularly the patellofemoral joint. Tibiofemoral angle was used to assess the tibiofemoral joint. Excessive Q angle or tibiofemoral angle can lead to patellofemoral pain syndrome, OA, or knee instability. Genu recurvatum was used to evaluate knee hyperextension that may cause ligament instability. Tibial torsion indicated external tibial rotation. Foot pronation was abnormal foot alignment that involved heel eversion, forefoot abduction, and internal rotation of leg. ${ }^{26}$

The prevalence of lower extremity malalignment in the general population has not yet been reported. However, lower extremity malalignment is related to lower extremity MSDs. Our study demonstrated that the prevalence of lower extremity malalignment was high. Similarly, Puntumetakul et $\mathrm{al}^{5}$ also reported that lower limb MSDs were highly prevalent among rice farmers. However, in that study, the findings showed the hip to be the joint with the highest prevalence of signs in the lower limb region (41\%), while in the current study our findings showed that the foot and knee had the highest prevalence of malalignment. This might be due to the difference in participant characteristics including age and BMI.

In particular, with regard to age, previous studies showed that older farmers may have increased articular damage and 
decreased physical performance. ${ }^{19,24}$ Age was a high risk factor for hip MSDs among farmers. ${ }^{12}$ The mean age of participants in the current study was 45 years, while Puntumetakul et $\mathrm{al}^{5}$ reported that the mean age of participants in their study was 54 years old and that most participants were older farmers (60-75 years old).

Additionally, previous studies have demonstrated that being overweight is associated with chronic lower extremity musculoskeletal symptoms. ${ }^{16}$ The number of overweight participants in the current study might account for the difference. In the current study, $30 \%$ of participants were overweight, while Puntumetakul et $\mathrm{al}^{5}$ reported that the number of overweight participants in their study was $41 \%$.

The current study found the highest prevalence of lower extremity disorder was foot and knee malalignment. This malalignment may arise from ergonomic factors associated with ankle and foot MSDs. Most rice farming tasks are performed with repetitive forward bending and twisting, heavy carrying or lifting, and prolonged standing or walking and include controlling heavy vibrating machinery while barefoot on slippery tilted surfaces. ${ }^{2}$ These conditions are risk factors for lower extremity MSDs that might result in increasing lower limb instability and injury. ${ }^{15}$ Moreover, ankle and foot malalignment might be due to adverse effects between ground reaction forces and rotational malalignment. Abnormal biomechanical effects usually occur on the weight-bearing surfaces during prolonged activities in the stance phase. The harvesting of rice cultivation involves prolonged walking on hard soil, ${ }^{2}$ which may increase the friction force to the feet and lead to chronic musculoskeletal symptoms. ${ }^{34}$ Other rice cultivation processes, specifically plowing and planting tasks, are performed in prolonged standing or walking barefoot on slippery uneven surfaces. These environmental conditions would challenge farmers to control leg alignment and may increase their ankle and foot instability, which could result in chronic ankle and foot musculoskeletal symptoms. ${ }^{15-21}$

Repetitive lower extremity motion in awkward postures may increase lower extremity discomfort and chronic musculoskeletal symptoms. ${ }^{6,7,21}$ Heavy lifting or carrying may overload legs, foot muscles, and tendons. ${ }^{6,7,21}$ Prolonged standing or walking can potentially increase the duration of compressive forces, increase fatigue, and lead to damage of leg and foot tissues ${ }^{6,7,14,15,21}$ Prolonged work-related risk factors could lead to progressive abnormal biomechanical function and result in increasing risk of chronic lower extremity musculoskeletal symptoms and malalignment. ${ }^{8,9}$ Therefore, rice cultivation tasks may increase lower limb MSDs and malalignment. Excessive foot pronation can lead to increased lower extremity strain and disorders, including compressive knee loading that results in lower limb MSDs, plantar fasciitis, stress fractures of the foot and tibia, medial tibial stress syndrome, and patellofemoral pain symptoms. ${ }^{34-36}$

Most farming tasks were performed in prolonged standing or walking, stooping, and twisting, which are associated with chronic knee musculoskeletal symptoms. This exposure may increase abnormal knee loading, lead to injury such as $\mathrm{OA}$ and patellofemoral pain syndrome, and might indicate knee malalignment. ${ }^{8} 9$ Moreover, farming activities for more experienced farmers involved walking with lower legs and feet immersed in muddy soil. This environment may lead to increased leg and knee pain arising from postural instability and fatigue. ${ }^{29}$ In addition, abnormal knee alignment may be caused by hip, ankle, or foot malalignment. Greater $\mathrm{Q}$ angle is associated with excessive femoral internal rotation, external tibial rotation, or tibiofemoral angle. ${ }^{8,9}$ Other lower extremity malalignment found in this study included external tibial torsion, anterior pelvic tilt, femoral antetorsion, and genu recurvatum. Prolonged exposure to physical work hazards can produce lower extremity malalignment. Lower extremity malalignment can lead to increased risk of MSDs. Anterior pelvic tilt might lead to increased lumbar lordosis, resulting in low back pain and hamstring muscle strain. ${ }^{37}$ Excessive femoral antetorsion and external tibial torsion can cause abnormal shear force loading on the knee joint and, therefore, lead to patellofemoral pain syndrome and knee OA. ${ }^{38}$ Genu recurvatum may result from knee ligament laxity, and can increase anterior tibia translation and anterior cruciate ligament injury. ${ }^{39}$

Lower extremity malalignment was associated with individual factors. Sex, age, BMI, and years farming were important risk factors for lower extremity malalignment. Sex (being female) was found to increase the risk of abnormal Q angle, tibiofemoral alignment, and genu recurvatum. Similarly, previous studies have shown that females have a greater $\mathrm{Q}$ angle, tibiofemoral malalignment, and genu recurvatum compared with males. ${ }^{17,18}$ Sex differences related to lower limb malalignment may be due to structure or function. ${ }^{17-20}$ Knee malalignment in females may result from an increased pelvic width, and internally rotated and valgus hips compared with males. ${ }^{18}$ Moreover, being female may decrease knee muscle strength and physical performance, which may lead to excessive abnormal joint loading and knee injury, resulting in knee malalignment. ${ }^{20,40}$ Females were almost 8.6 times more likely to demonstrate genu recurvatum. It is possible that females have greater knee ligament laxity than males, and therefore females have a greater risk of genu recurvatum than 
males. ${ }^{41}$ Being female was a risk factor for femoral antetorsion. The finding related to femoral antetorsion appears to be conflicting. Previous studies have reported that females have increased femoral antetorsion compared with males. ${ }^{17,18}$ However, there is a lack of clarity as to why females had greater hip antetorsion than males in the present study.

Obesity is one of the important risk factors for lower extremity malalignment. Being overweight is a risk factor for developing abnormal pelvic tilt, Q angle, and tibiofemoral angle. Overweight farmers presented with anterior pelvic tilt 8.6 times more frequently than normal weight farmers. Viester et $\mathrm{al}^{16}$ found similar results in their study, namely, that anterior pelvic tilt increased with greater body weight. Increased abdominal circumference may increase lumbar lordosis and hence anterior pelvic tilt. ${ }^{41}$ Being overweight was also a risk factor for excessive $\mathrm{Q}$ angle and tibiofemoral angle, leading to excess stress on the knee joint.

Our results indicate that age is an important risk factor for limb length inequality. Aging reportedly increases the rate of degeneration and articular damage, which may affect lower limb repetitive injury and anatomical alignment adaptation. ${ }^{19,24}$ In addition, a functional limb length inequality may result from compensatory asymmetric joint motion, adaptive imbalance of soft tissue, or ligament laxity. ${ }^{36}$ Aging may decrease lower limb muscle strength and physical performance, and lead to greater lower limb ligament laxity, resulting in a limb length inequality. ${ }^{19,24}$

Years of farming were associated with abnormal foot and knee alignment. This may be due to work-related risk factors. Farming activities with high ergonomic risk factors may increase chronic lower extremity musculoskeletal symptoms. ${ }^{6,7}$ Farmers who perform work activities in extreme environmental conditions may find it difficult to maintain neutral postures, and this may affect alignment adaptation. ${ }^{6,7}$ Lower extremity malalignment may occur in experienced rice farmers due to the high physical workload associated with excessive knee joint and foot loading. This exposure was found to be associated with degenerative joints, a risk factor for malalignment. ${ }^{6,7}$ Additionally, previous studies have revealed that more experienced farmers reported more leg pain due to increased body instability during the foot push-off phase of walking in muddy soil. Farming activities performed with high leg and foot exertion may lead to fatigue and chronic musculoskeletal symptoms. ${ }^{4}$ Moreover, our study found that farming was related to lower extremity malalignment. However, participants with abnormal malalignment did not report work absence, and they managed pain relief with medication such as analgesics or they would take rests $2-3$ times daily for up to 2-3 hours. Further studies should be conducted to explore work absence in farmers with lower extremity malalignment to enhance our understanding of the effects on work in farmers with lower extremity malalignment.

This study is the first to describe the prevalence, and identify risk factors, of lower extremity malalignment among Thai rice farmers. The prevalence of lower extremity malalignment in the general population has not been reported, and was not investigated in the current study. Therefore, our prevalence results of lower extremity malalignment were not compared with that in the general population. Further research should be conducted into the prevalence of lower extremity malalignment in the general population and in other workers' groups. A comparison of the prevalence of lower extremity malalignment in rice farmers with that of nonlaborers would have strengthened our findings.

Our results indicate that sex and age are risk factors for lower extremity abnormal alignment in rice farmers. A further study with a control group matched for sex and age would help to determine additional risk factors for lower extremity malalignment.

The current study focused on identifying factors related to individual characteristics, working hours, and years of farming experience. Previous studies have not reported years of experience or daily working hours as variables related to lower extremity malalignment. This study demonstrated a trend toward a change in the degree of lower extremity malalignment angle for each additional year of farming experience. However, further studies could investigate discrete periods of experience or varying amounts of daily work hours.

Other risk factors for lower extremity malalignment were not considered. The results from the present study could not confirm whether any ergonomic risk factors (such as force, motion and repetition) were associated with lower extremity malalignment. The results of this study should be considered in approaches to improve occupational health and safety in rice farming. Future prospective epidemiological studies on other risk factors for lower extremity malalignment should be identified to guide the development of occupational health and safety programs in order to reduce and prevent lower extremity malalignment.

\section{Conclusion}

In summary, signs of foot and knee malalignment were highly prevalent in rice farmers. Sex, age, BMI, and years of experience were important factors related to lower extremity malalignment. Being female was shown to be a major risk factor for abnormal Q angle, tibiofemoral angle, and genu 
recurvatum. In contrast, being female was not associated with femoral antetorsion. Being overweight was found to be the major risk factor for abnormal pelvic tilt, $\mathrm{Q}$ angle, and tibiofemoral angle malalignment. Aging was found to be a significant risk factor for limb length inequality. More years of farming experience was a risk factor of abnormal Q angle, tibiofemoral angle, and foot malalignment.

These findings reinforce the need for clinicians to consider the relationship between occupational activities and lower extremity malalignment. Consideration of preventive strategies to reduce injury risk factors in rice farming may be useful. Health education about ways to control body weight, and discussions about work characteristics including work duration may be worthwhile.

\section{Acknowledgments}

The authors thank the participants in Khon Kaen Province. This study was supported by grants from Thailand Research Fund (TRF), Office of the Higher Education Commission (OHEC), and Khon Kaen University (No MRG5680009). The opinions expressed in this article are those of the authors and do not necessarily reflect the views of TRF, OHEC, or Khon Kaen University. We also thank the Research Center in Back, Neck, Other Joint Pain and Human Performance (BNOPH) for partial support of this study.

\section{Disclosure}

The authors report no conflicts of interest in this work.

\section{References}

1. Thai Rice Exporters Association. Rice Export Quantity and Value: 2014. Online sources 2014 [cited January 28, 2015]. Available from: http:// www.thairiceexporters.or.th/statistic_2014.html. Accessed January 29, 2014.

2. Mokkamul P. Ethnobotanical study of rice growing process in northeastern Thailand. Ethnobotany Res Appl. 2006;4:213-222.

3. Puntumetakul R, Yodchaisarn W, Emasithi A, Keawduangdee P, Chatchawan U, Yamauchi J. Prevalence and individual risk factors associated with clinical lumbar instability in rice farmers with low back pain. Patient Prefer Adherence. 2015;9:1-7.

4. Swangnetr M, Kaber D, Puntumetakul R, Gross MT. Ergonomics-related risk identification and pain analysis for farmers involved in rice field preparation. Work. 2014;49(1):63-71.

5. Puntumetakul R, Siritaratiwat W, Boonprakob Y, Puntumetakul M. Prevalence of musculoskeletal disorder in farmer: case study in Sila, Muang Khon Kaen, Khon Kaen Province. J Med Tech Phys Ther. 2011;23: 298-303.

6. Reid CR, Bush P, Karwowski W, Durrani S. Occupational postural activity and lower extremity discomfort: a review. Int J Ind Ergonom. 2010;40:247-256.

7. Jaffer N, Abdul-Tharim AH, Mohd-Kamar IF, Lop NS. A literature review of ergonomics risk factors in construction industry. Proceeding of the 2nd International Building Control Conference. Procedia Eng. 2011;20:89-97.
8. Daneshmandi H, Saki F, Shahheidari S, khouri A. Lower extremity malalignment and its linear relation with $\mathrm{Q}$ angle in female athletes. Procedia Soc Behav Sci. 2011;15:3349-3354.

9. Shultz SJ, Nguyen A, Levine BJ. The relationship between lower extremity alignment characteristics and anterior knee joint laxity. $J$ Athl Train. 2009;1:54-60.

10. Solberg G. Postural Disorder and Musculoskeletal Dysfunction: Diagnosis, Prevention and Treatment. 2 nd ed. China: Churchill Livingstone; 2008.

11. Suri P, Morgenroth DC, Hunter DJ. Epidemiology of osteoarthritis and associated comorbidities. PMR. 2012;4(5 Suppl):S10-S19.

12. Power CM, Chen PY, Reischl SF, Perry J. Comparison of foot pronation and lower extremity rotation in persons with and without patellofemoral pain. Foot Ankle Int. 2002;23(7):634-640.

13. Griffin LY. Understanding and prevention noncontact anterior cruciate ligament injuries. Am J Sport Med. 2006;34:1512-1531.

14. Osborne A, Blake C, Fullem BM, et al. Risk factors for musculoskeletal disorders among farm owners and farm workers: a systematic review. Am J Ind Med. 2012;55:376-389.

15. Yu S, Lu M, Gu G, Zhou W, He L, Wang S. Musculoskeletal symptoms and associated risk factors in a large sample of Chinese workers in Henan province of China. Am J Ind Med. 2012;55: 281-293.

16. Viester L, Verhagen EA, Hengel KM, Koppes L, van der Beek AJ. The relation between body mass index and musculoskeletal symptoms in the working population. BMC Musculoskelet Disord. 2013;14:238.

17. McKeon JM, Hertel J. Sex differences and representative values for 6 lower extremity alignment measures. J Athl Train. 2009;44(3): 249-255.

18. Nguyen AD, Shultz SJ. Sex differences in clinical measures of lower extremity alignment. J Orthop Sports Phys Ther. 2007;37(7): 389-398.

19. Tamari K, Tinley P, Briffa K, Aoyagi K. Ethnic-, gender-, and agerelated differences in femoral antetorsion, and tibiofibular torsion: crosssectional study among healthy Japanese and Australian caucasians. Clin Anat. 2006;19:59-67.

20. Keogh E, Herdenfeldt M. Gender, coping and the perception of pain. Pain. 2002;97:195-201.

21. Messing K, Tissot F, Stock S. Distal lower-extremity pain and work postures in the Quebec population. Am J Public Health. 2008;98: 705-713.

22. Zyroul R, Hossain MG, Abbas AA, Kamarul T. Knee laxity of Malaysian adults: gender differentials, and association with age and anthropometric measures. Knee. 2014;21(2):557-562.

23. Murphy DF, Connolly DA, Beynnon BD. Risk factors for lower extremity injury: a review of the literature. Br J Sports Med. 2003;37: $13-29$.

24. Brady RJ, Dean JB, Skinner TM, Gross MT. Limb length inequality: clinical implications for assessment and intervention. J Orthop Sports Phys Ther. 2003;33:221-234.

25. Leard JS, Crane BA, Ball KA. Intrarater and interrater reliability of 22 clinical measures associated with lower quarter malalignment. J Manipulative Physiol Ther. 2009;32:270-276.

26. Magee DJ. Orthopedic Physical Assessment. Philadelphia, PA: WB Saunders; 2006.

27. Shultz SJ, Nguyen A, Windley TC, Kulas AS, Botic TL, Beynnon BD. Intratester and intertester reliability of clinical measures of lower extremity anatomical characteristics; implications for multi-center studies. Clin J Sport Med. 2006;16:155-161.

28. Herrington L. Assessment of the degree of pelvic tilt within a normal asymptomatic population. Man Ther. 2011;16:646-648.

29. Nguyen AD, Shultz SJ. Identifying relationships among lower extremity alignment characteristics. J Athl Train. 2009;44:511-518.

30. Devan M, Pescatello L, Faghrit P, Anderson J. A prospective study of overuse knee injuries among females athletes with muscle imbalances and structural abnormalities. J Athl Train. 2004;39:263-267. 
31. Fouilleron N, Marchetti E, Autissier G, Gougeon F, Migaud H, Girard J. Proximal tibialderotation osteotomy for torsional tibial deformities generating patello-femoral disorders. Orthop Traumatol Surg Res. 2010;96(7):785-792.

32. Jonson SR, Gross MT. Intraexaminer reliability,interexaminer reliability, and mean values for nine lower extremity skeletal measures in healthy naval midshipmen. J Orthop Sports Phys Ther. 1997;25:253-263.

33. Hosmer DW, Lemeshow S. Applied Logistic Regression. 2nd ed. New York, NY: John Wiley and Sons; 2000.

34. Barnes A, Wheat J, Milner C. Association between foot type and tibial stress injuries: a systematic review. Br J Sports Med. 2008;42:93-98.

35. Yates B, White $\mathrm{S}$. Incidence and risk factors in the development of medial tibial stress syndrome among naval recruits. Am J Sports Med. 2004; 32(3):772-780.

36. Levinger $P$, Gilleard $W$. Tibia and rearfoot motion and ground reaction forces in subjects with patellofemoral pain syndrome during walking. Gait Posture. 2007;25(1);2-8.
37. Panayai S. The need for lumbar-pelvic assessment in the resolution of chronic hamstring strain. J Bodyw Mov Ther. 2010;14:294-298.

38. Krackow K, Mandeville D, Rachala S, Bayers-Thering M, Osterning L. Torsion deformity and joint loading for medial knee osteoarthritis. Gait Posture. 2011;33;625-629.

39. Terauchi M, Hatayama K, Yanagisawa S, Saito K, Takagishi K. Sagittal alignment of the knee and its relationship to noncontact anterior cruciate ligament injuries. Am J Sports Med. 2011;39:1090-1094.

40. Scerpella TA, Stayer TJ, Makhuli BZ. Ligamentous laxity and noncontact anterior cruciate ligament tear: a sex-based comparison. Orthopedics. 2005;28:656-660.

41. Vismara L, Menegoni F, Zaina F, Galli M, Negrini S, Capodaglio P. Effect of obesity and low back pain on spinal mobility: a cross sectional study in women. J Neuroeng Rehabil. 2010;7:3.

\section{Publish your work in this journal}

Patient Preference and Adherence is an international, peer-reviewed, open access journal that focuses on the growing importance of patient preference and adherence throughout the therapeutic continuum. Patient satisfaction, acceptability, quality of life, compliance, persistence and their role in developing new therapeutic modalities and compounds to optimize clinical outcomes for existing disease states are major areas of interest for the journal. This journal has been accepted for indexing on PubMed Central. The manuscript management system is completely online and includes a very quick and fair peer-review system, which is all easy to use. Visit http://www. dovepress.com/testimonials.php to read real quotes from published authors.

Submit your manuscript here: http://www.dovepress.com/patient-preference-and-adherence-journal 